\title{
A Rare Case of Diffuse Subependymal Periventricular Metastases from Small Cell Lung Carcinoma
}

\author{
Cong Thao Trinh ${ }^{a}$ Thanh Tam Thi Nguyen ${ }^{b}$ Hoang Anh Thi Van ${ }^{c}$ \\ Van Trung Hoang ${ }^{c}$

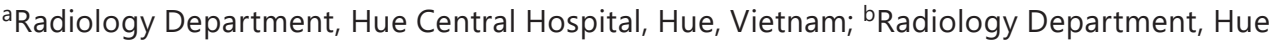 \\ University of Medicine and Pharmacy, Hue, Vietnam; 'Radiology Department, Thien Hanh \\ Hospital, Buon Ma Thuot, Vietnam
}

\section{Keywords}

Brain metastases - Brain tumor · Lung cancer - Small cell lung cancer - Subependymal periventricular metastases

\begin{abstract}
Small cell lung cancer, whose essence is neuroendocrine tumors, makes up proximately 14$20 \%$ of all lung cancer circumstances. Compared to non-small cell lung cancer, its clinical manifestation seems more positive and has a tendency to disseminate earlier in the process of its natural past. About $10 \%$ of patients present with brain metastases at the time of provisional diagnosis and sometimes all along the course of their disease, there will be $40-50 \%$ of developed brain metastases in addition. Although metastases in the brain parenchyma are often found in patients with advanced lung cancer, periventricular metastases are rare. We report one case of diffuse subependymal periventricular metastases from small cell carcinoma of the lung.

\section{Introduction}

Small cell lung cancer (SCLC), the most prevalent configuration of neuroendocrine lung cancer, has the highest speed of growth and early metastatic dispersion [1, 2]. At the time of confirmed diagnosis, the liver, bone, brain, and lung seem to be the four most common sites of metastasis in SCLC. Although parenchymal metastases are the most typical cases of brain

Cong Thao Trinh

Radiology Department, Hue Central Hospital

16 Le Loi Street

Hue 530000 (Vietnam)

thaotrinh2911@gmail.com
Van Trung Hoang

Radiology Department, Thien Hanh Hospital

17 Nguyen Chi Thanh Street

Buon Ma Thuot 630000 (Vietnam)

dr.hoangvantrungradiology@gmail.com 


\section{Case Reports in Oncology}
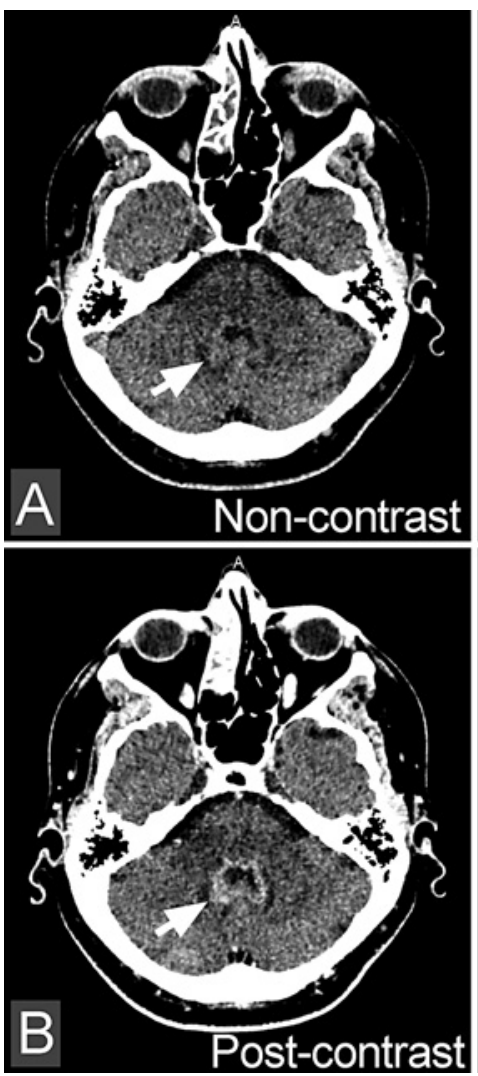

\begin{tabular}{l|l}
\hline Case Rep Oncol 2020;13:1304-1310 \\
\hline DOI: 10.1159/000508828 & $\begin{array}{l}\text { @ 2020 The Author(s). Published by S. Karger AG, Basel } \\
\text { www.karger.com/cro }\end{array}$ \\
\hline
\end{tabular}

Trinh et al.: Diffuse Subependymal Periventricular Metastases from Small Cell Lung Carcinoma
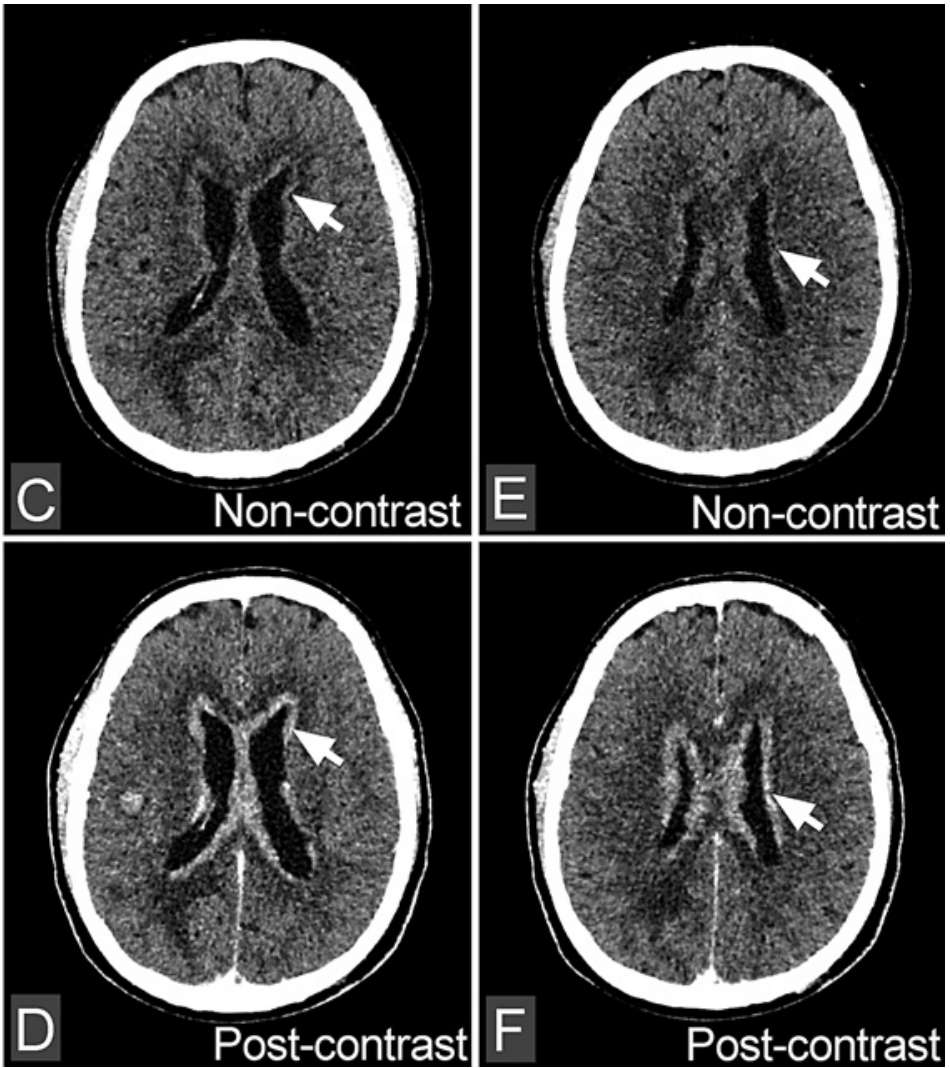

Fig. 1. Diffuse subependymal periventricular metastases from small cell carcinoma of the lung. A, C, E Noncontrast-enhanced CT images reveal diffuse subependymal periventricular, ribbon-like, dense areas (arrows). B, D, F Contrast-enhanced CT images show diffuse subependymal periventricular enhancement in bilateral (arrows).

metastases, diffuse subependymal periventricular metastases may also be found despite the rarity [3-5]. We report a case of a 61-year-old man with a history of lung cancer and currently diagnosed as diffuse subependymal periventricular metastases. The aim of this paper is to provide several characteristic imaging features of subependymal periventricular metastases in particular and brain metastases in general. Besides, we further present a few points about the clinical manifestations, imaging, treatment, and prognosis of brain metastases in patients with SCLC.

\section{Case Report}

A 61-year-old man, who had a background of hypertension and a heavy smoking history, had been diagnosed with small cell carcinoma of the right upper lobe of the lung graded as cT2aN3M1b with hepatic metastases about more than 1 year ago. He had received a cycle of cisplatin and carboplatin etoposide concomitant with radiotherapy. Approximately a year after treatment, he had symptoms of seizures and behavior problems. The neurological examination determined memory disturbances, paralysis, dysarthria, and bilateral extensor plantar responses. The patient had undergone computed tomography (CT) of the brain with contrast. Non-contrast-enhanced CT images revealed diffuse subependymal periventricular, 

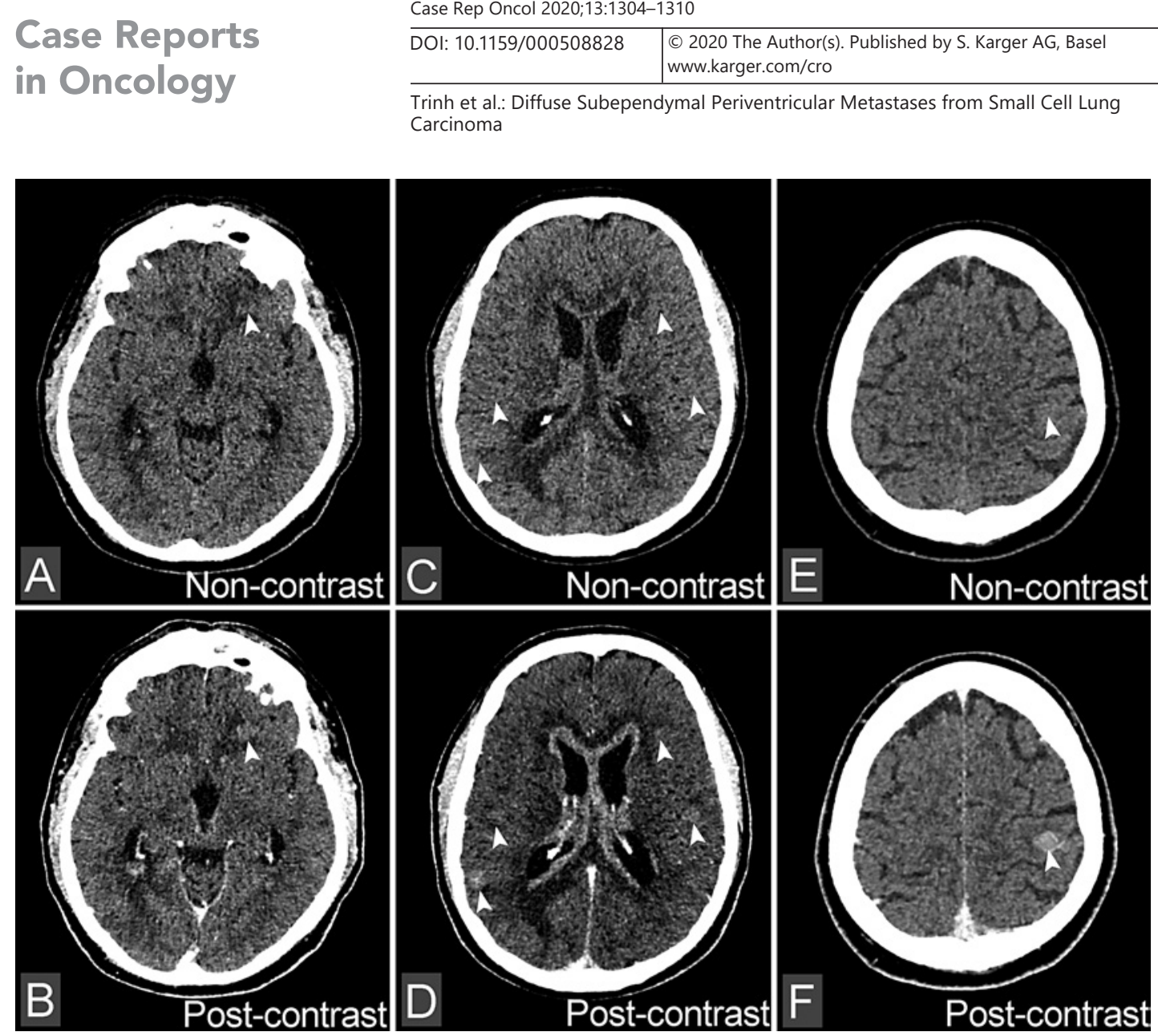

Fig. 2. A, C, E Non-contrast-enhanced CT images cannot detect lesions of metastasis. B, D, F Contrast-enhanced CT images show enhancement of nodules scattered in the brain parenchyma (arrows) mainly positioning in the white matter and gray matter boundaries with discreet edema around the lesion.

ribbon-like, dense areas. These hyperdense areas showed vivid, homogenous post-administration of contrast agents (Fig. 1). In addition, CT images disclosed few hyperdense nodular lesions scattered in the bilateral brain parenchyma (Fig. 2). There were signs of edema around the lesions (Fig. 3). Periventricular tumor infiltration was also another useful feature for diagnosing diffuse subependymal periventricular metastases. A picture illustrating the summary of the lesions of this case was also introduced (Fig. 4).

\section{Discussion}

Brain metastases are encountered as more than half of intracranial tumors. They mainly originate from lung cancer, malignant melanoma, breast cancer, renal cell cancer, and gastrointestinal cancer [4-7]. Intracranial metastases tend to distribute in the supratentorial region; only about one-third of cases are located in the infratentorial region. The most common affected location is the junction between the white and gray matter also called the "watershed area," especially at the border between the middle and the posterior cerebral arterial territories [7]. The neurological signs and symptoms are not specific to the disease but related to 


\section{Case Reports in Oncology}
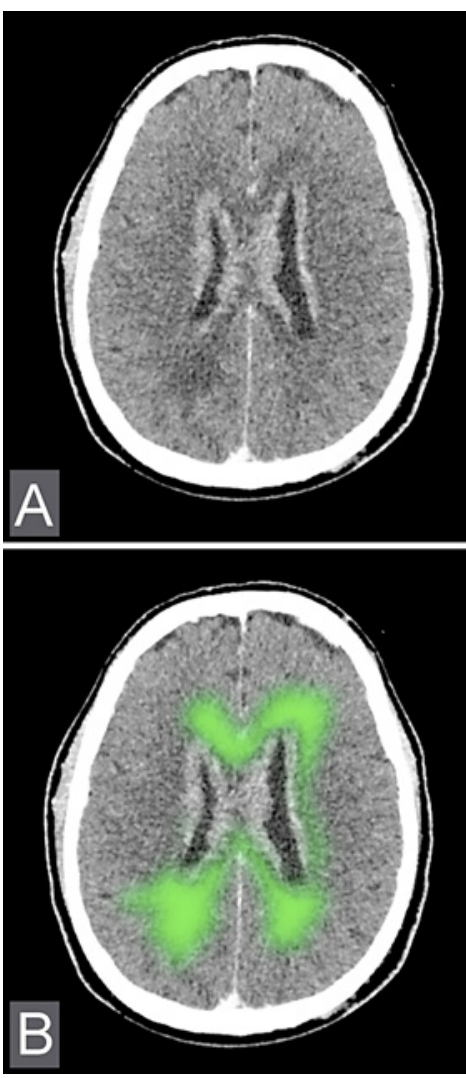
www.karger.com/cro

Trinh et al.: Diffuse Subependymal Periventricular Metastases from Small Cell Lung Carcinoma
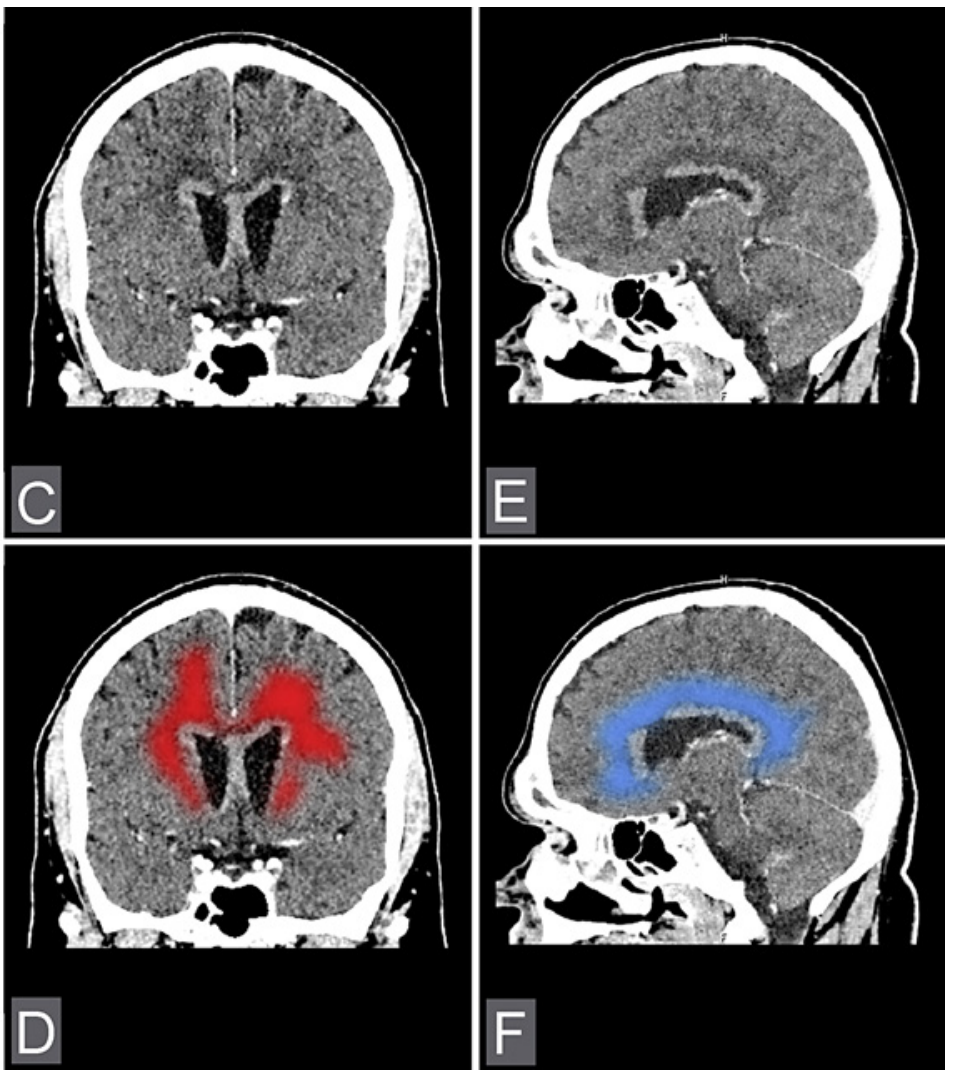

Fig. 3. A, C, E Axial, coronal, and sagittal CT images show edema of the brain around the lesions. B, D, F The illustration of edema areas surrounding metastasis lesions.

the location and number of metastatic lesions. Moreover, the severity of these symptoms may reflect the degree of tumor-related vasogenic edema. The common signs and symptoms include headache, numbness, altered sensorium, and seizures [8]. SCLC is more likely to be associated with multiple brain metastases distributed in the cerebellum and frontal lobe [9]. However, metastases in the periventricular area are very rare. The diagnosis of brain metastases needs to be based on the history of the original lung tumor, clinical presentation, laboratory test, and diagnostic imaging. Imaging of the brain is a prerequisite for the patients with SCLC who are suspected of having brain metastases because metabolic abnormalities or paraneoplastic syndromes can cause the symptoms that mimic those caused by intracranial metastases $[4,6,10]$.

Non-contrast-enhanced CT image is difficult to identify metastases unless the mass is very large. White matter edema is a suggestive clue. Nevertheless, it may be confused with other conditions such as cerebral infarction or white matter disease. With diffuse subependymal periventricular metastases, contrast-enhanced CT image is a better manner to detect a typical ribbon of high density along with the ventricular system with vivid contrast enhancement. Magnetic resonance imaging (MRI) is the key imaging modality used in the evaluation of brain metastases. On T2-weighted images, tumors show a moderate increased signal intensity, peritumoral edema reveals high-intensity signal. The most helpful imaging study is MRI with the administration of gadolinium. Brain metastases from SCLC usually occur at multiple sites and tend to be located in the posterior cranial fossa [9]. This is better visualized on MRI. It will help to detect small lesions that might be overlooked on CT scans. 

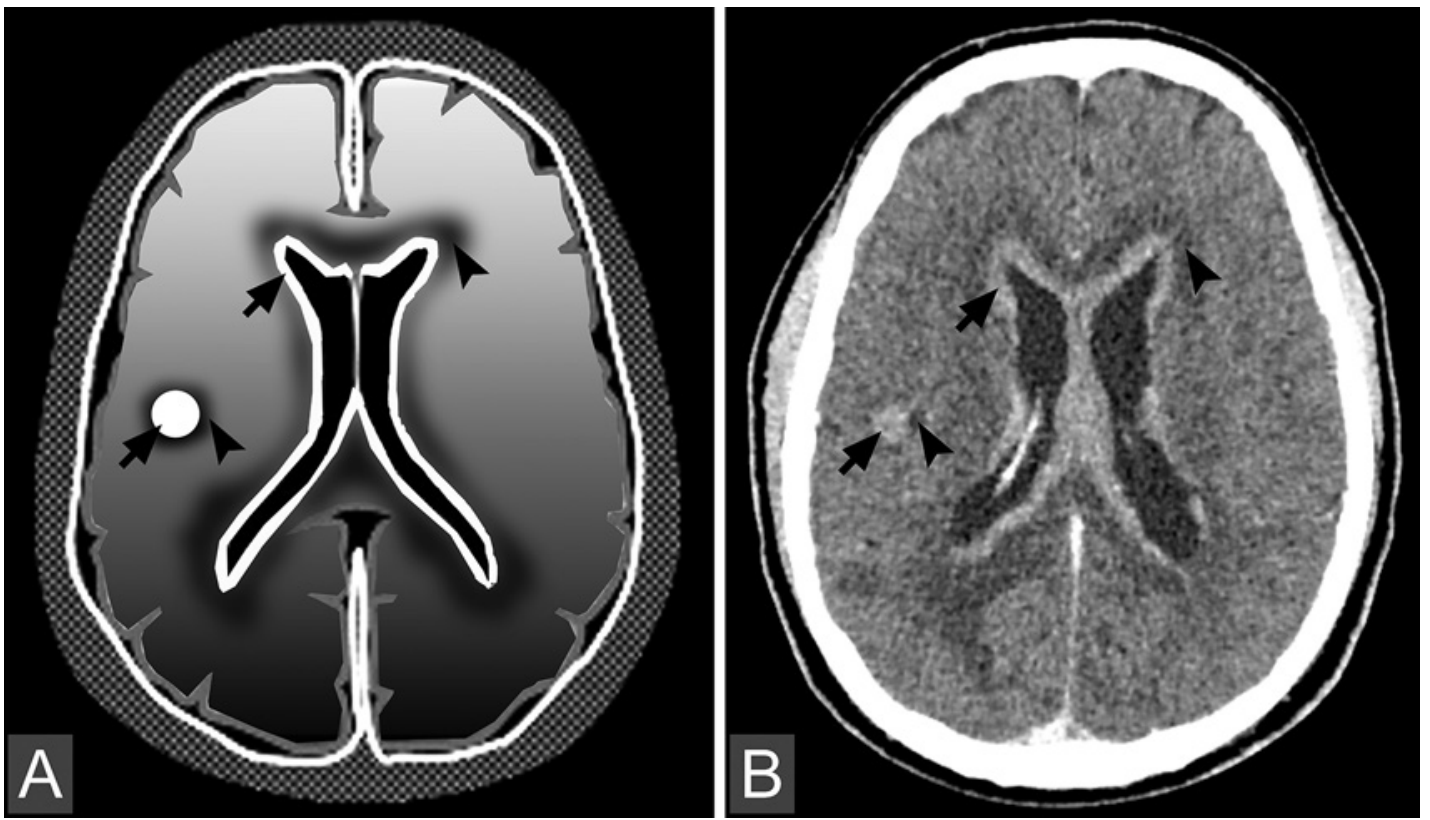

Fig. 4. Illustration (A) and axial CT image (B) present metastasis lesions in the form of nodules in the parenchyma and ribbons in periventricular regions (arrows). Note that the edema in brain metastases is usually a peritumoral vasogenic edema (arrowheads). As for edema of white matter, the typical sign is a finger glove edema.

Moreover, MRI is useful to identify leptomeningeal involvement or subependymal periventricular metastases, an uncommon finding in patients with SCLC. It usually appears as diffuse or multiple periventricular subependymal foci, mainly iso-intense on T1-weighted and on T2-weighted images compared with gray matter. The characteristic imaging features of subependymal metastases after contrast agent administration is ribbon-like of high intensity with vivid enhancement. Moreover, diffusion images demonstrate the intracranial tumors which present high signal in diffusion-weighted imaging, and low apparent diffusion coefficient values are more probably associated with SCLC brain metastases [3]. In addition to establishing a diagnosis, MRI can also be used in treatment planning and as a baseline for evaluating response to treatment [11].

In our case with histologically confirmed small cell carcinoma arising from the lung, the neurological manifestations have appeared 1 year after the initial diagnostic. CT images show the tumor involved in the ventricular system that represents diffuse periventricular infiltration forming nodules protruding into the ventricles and few intraparenchymal nodular metastases were found. The diagnosis of the diffuse cerebral subependymal metastases from SCLC is appropriate for this case. In the literature, some cases have been reported with diffuse subependymal periventricular metastases originated from melanoma malignancy or lung oat cell carcinoma. Metastases in the periventricular area as in our case are very rarely reported and the pathobiological mechanisms remand unclear [9, 12].

Non-contrast-enhanced CT images are insufficient to identify metastases unless the mass is very large. White matter edema is a suggestive clue. With diffuse subependymal periventricular metastases, contrast-enhanced CT image is a better manner to detect a typical ribbon of high density along with the ventricular system with strong contrast enhancement. In our patient, a non-contrast-enhanced CT image shows a typical sheet of hyperdensity along the ventricular system but in other previous cases in the literature, a non-contrast-enhanced CT 
image only reveals normal or dilatation of ventricular cavities [3]. Thin periventricular dense area or small intraventricular nodular masses may be confused with other conditions such as inflammatory processes, subependymal spreading lesions, or white matter disease [12]. MRI is the key imaging modality used in the evaluation of brain metastases [13], but our patient does not undergo brain MRI because the information on CT is very clear.

Although periventricular metastases are an uncommon place from SCLC, the therapy of treatment in this category is also based on methods similar to metastases in the brain generally. Treatment of brain metastases from lung cancer requires a combination of methods. In the cases of brain metastases, treatment and intervention are indispensable to limit progressive nerve damage. However, methods of treatment for brain metastases have not been completely clarified. It depends on the experience of an oncologist to choose the best method for the patient. Some main therapies for treating brain metastases from SCLC are currently applied such as whole-brain radiation therapy, stereotactic radiosurgery, brachytherapy, systemic chemotherapy, surgery, or immunotherapy [4, 14, 15]. Besides, the therapy antiepileptic medications and corticosteroids are used for symptomatic treatment and aim to control intracranial pressure. The effect of corticosteroids is to decrease the brain-to-tumor capillary permeability, thereby reducing cerebral edema. Antiepileptic drugs are used when the patient exhibits seizures, such as phenytoin, but must always be considered.

Although patients with brain metastases from lung cancer have a poor prognosis overall, certain factors have been identified that are predictive of an improved outcome $[3,9,10]$. Age, performance status, control of the primary tumor, and the presence or absence of extracranial metastatic disease were found to significantly improve survival. Few reports have shown an extended survival in SCLC patients with solitary brain metastasis who have received radiotherapy and chemotherapy. These patients remain disease-free after 2-3 years of follow-up. However, other studies have reported that the survival rates of the patients with brain metastasis were still dismal with a median survival rate of 9 months [7-10].

\section{Conclusion}

Subependymal periventricular metastases are not common. This type of metastases usually originates from primary types of organ cancer. It arises from lung cancer that is very rare. Therefore, when a patient has a history of lung cancer, we must carefully consider whether metastases around the ventricles are present or not, to avoid missing out metastases. A CT and MRI with contrast are the best choices to check if central nervous system metastases exist. Patients with brain metastases from lung cancer have a poor prognosis overall. Consequently, the treatment and prognosis of brain metastases from lung cancer require a combination of methods.

\section{Statement of Ethics}

The authors declare that ethics approval was not required for this case report. Written informed consent for the publication of the patient's clinical details and images was obtained from the patient.

\section{Conflict of Interest Statement}

There are no conflicts of interest to declare.

\section{Karger'}




\section{Case Reports in Oncology}

\begin{tabular}{l|l}
\hline Case Rep Oncol 2020;13:1304-1310 \\
\hline DOI: 10.1159/000508828 & $\begin{array}{l}\text { @ 2020 The Author(s). Published by S. Karger AG, Basel } \\
\text { www.karger.com/cro }\end{array}$ \\
\hline
\end{tabular}

Trinh et al.: Diffuse Subependymal Periventricular Metastases from Small Cell Lung Carcinoma

\section{Funding Sources}

The authors did not receive any funding.

\section{Author Contributions}

The authors contributed equally.

\section{References}

1 Koinis F, Kotsakis A, Georgoulias V. Small cell lung cancer (SCLC): no treatment advances in recent years. Transl Lung Cancer Res. 2016 Feb;5(1):39-50.

2 Kalemkerian GP, Akerley W, Bogner P, Borghaei H, Chow LQ, Downey RJ, et al. National Comprehensive Cancer Network. Small cell lung cancer. J Natl Compr Canc Netw. 2013 Jan 1;11(1):78-98.

3 Vannier A, Gray F, Gherardi R, Marsault C, Degos J, Poirier J. Diffuse subependymal periventricular metastases. Report of three cases. Cancer. 1986 Dec 15;58(12):2720-5.

4 Chi A, Komaki R. Treatment of brain metastasis from lung cancer. Cancers (Basel). 2010 Dec 15;2(4):2100-37.

5 D’Antonio C, Passaro A, Gori B, Del Signore E, Migliorino MR, Ricciardi S, et al. Bone and brain metastasis in lung cancer: recent advances in therapeutic strategies. Ther Adv Med Oncol. 2014May;6(3):101-14.

6 Pekmezci M, Perry A. Neuropathology of brain metastases. Surg Neurol Int. 2013 May 2;4(Suppl 4):S245-55.

7 Saha A, Ghosh SK, Roy C, Choudhury KB, Chakrabarty B, Sarkar R. Demographic and clinical profile of patients with brain metastases: A retrospective study. Asian J Neurosurg. 2013 Jul;8(3):157-61.

8 Rodin D, Banihashemi B, Wang L, Lau A, Harris S, Levin W, et al. The Brain Metastases Symptom Checklist as a novel tool for symptom measurement in patients with brain metastases undergoing whole-brain radiotherapy. Curr Oncol. 2016 Jun;23(3):e239-47.

9 Wang G, Xu J, Qi Y, Xiu J, Li R, Han M. Distribution Of Brain Metastasis From Lung Cancer. Cancer Manag Res. 2019 Nov 1;11:9331-8.

10 Lekic M, Kovac V, Triller N, Knez L, Sadikov A, Cufer T. Outcome of small cell lung cancer (SCLC) patients with brain metastases in a routine clinical setting. Radiol Oncol. 2012 Mar;46(1):54-9.

11 Zakaria R, Das K, Radon M, Bhojak M, Rudland P, Sluming V, et al. Diffusion-weighted MRI characteristics of the cerebral metastasis to brain boundary predicts patient outcomes. BMC Med Imaging. 2014 Aug 3;14:26.

12 Kawahara I, Fujimoto T, Ozono K, Hirose M, Toyoda K, Kitagawa N, et al. [A Very Rare Case of Diffuse Subependymal Metastases from Small Cell Lung Carcinoma]. Neurol Surg. 2017 Feb;45(2):167-74.

13 Pope WB. Brain metastases: neuroimaging. Handb Clin Neurol. 2018;149:89-112.

14 Owonikoko TK, Arbiser J, Zelnak A, Shu HK, Shim H, Robin AM. et al. Current approaches to the treatment of metastatic brain tumours. Nat Rev Clin Oncol. 2014 Apr 11;4:203-22.

15 Steindl A, Schlieter F, Klikovits T, Leber E, Gatterbauer B, Frischer J, et al. Prognostic assessment in patients with newly diagnosed small cell lung cancer brain metastases: results from a real-life cohort. J Neurooncol. 2019 Oct;145(1):85-95. 